\title{
Matéria
}

ISSN 1517-7076

\section{Estudo da viabilidade de utilização de fibras naturais curtas em matrizes de resina epóxi}

\author{
Martins, R.R. ${ }^{\text {I; }}$ Pires, A.T.N. ${ }^{\mathrm{II}}$; Al-Qureshi, H.A. ${ }^{\mathrm{I}}$; Barra, G.M.O. ${ }^{\mathrm{I}}$

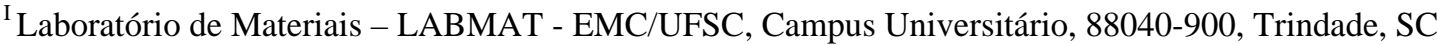 \\ e-mail: rach1d@pop.com.br, alhazim@emc.ufsc.br, guiga@emc.ufsc.br \\ ${ }^{\text {II }}$ Grupo de Estudo em Materiais Poliméricos - POLIMAT - QMC/UFSC, Campus Universitário, 88040-900, \\ Trindade, SC \\ e-mail: pires@qmc.ufsc.br
}

\section{RESUMO}

Fibras curtas de origem mineral, constituídas, predominantemente, de sílica amorfa, foram caracterizadas e avaliadas quanto a sua viabilidade de utilização como agente de reforço em matrizes de resina epóxi. As fibras naturais de sílica amorfa, denotadas por FNSA, foram incorporadas em matrizes de resina epóxi e as propriedades mecânicas avaliadas através de ensaios de tração. Foi estudada a influência do tratamento superficial da FNSA com agente aminossilano (AS), nas propriedades micro e macroscópicas dos compósitos, e comparado o comportamento mecânico dos compósitos obtidos com fibras naturais de sílica amorfa e fibras de vidro moídas (FV) comerciais. Os resultados sugerem que as fibras de sílica amorfa podem substituir fibras de vidro moídas, as quais vêm sendo utilizadas como agente de reforço em matriz de resina epóxi, uma vez que as propriedades mecânicas dos compósitos foram comparáveis ou superiores aos compósitos reforçados com fibras de vidro moídas.

Palavras-chaves: fibras naturais de sílica amorfa, fibra de vidro, resina epóxi, compósitos.

\section{Properties of natural amorphous silica fibers as epoxy resin reinforcement}

\section{ABSTRACT}

A novel kind of short natural fiber obtained from mineral resources, which consists mainly of pure amorphous $\mathrm{SiO}_{2}$, was characterized and applied as a reinforcing agent in epoxy resin. Natural amorphous silica fibers (NASF) were incorporated in epoxy resin and the obtained composites were mechanically tested. The effect of the treatment of NASF chemically modified with aminosilane as well as the aspect ratio of the fibers on the mechanical properties of the composite were evaluated and compared with properties obtained with FNSA without treatment. The results obtained show that these natural fibers are potential candidates for the substitution of short glass fibers as a reinforcement phase in polymer composites, since the mechanical properties obtained were in all cases similar or superior to the ones obtained with commercial short glass fibers.

Keywords: natural amorphous silica fibers, epoxy resin, short glass fiber, polymer composites.

\section{INTRODUÇÃO}

O desenvolvimento de novos processos, buscando a obtenção de materiais com aplicações específicas, tem sido de grande interesse, nos últimos anos. Os materiais poliméricos vêm ocupando um espaço cada vez maior nos setores tecnológicos, pela possibilidade de substituição aos materiais convencionais, tais como: metais, madeira e vidro. Entretanto, a aplicação de alguns polímeros fica limitada pelas características e desempenho mecânico, tais como baixa resistência ao impacto, à tração e à fadiga, estabilidade dimensional e resistência à abrasão. A adição de aditivos ou fibras de diferentes fontes tem sido uma alternativa para a obtenção de materiais com propriedades específicas e diferentes dos componentes puros. As propriedades de polímeros reforçados com fibras são dependentes da proporção dos constituintes da mistura e da geometria da fase dispersa, bem como da distribuição, comprimento, orientação e forma das 
fibras e adesão da interface matriz/fibra. A adesão interfacial matriz/fibra pode ser melhorada com o uso de agentes de acoplamento que modificam a superfície da fibra, reduzindo a energia interfacial [1].

A preocupação mundial com o meio ambiente e a sobrevivência das indústrias nacionais em um mercado competitivo vêm incentivando a utilização de materiais naturais, tendo as fibras naturais despertado interesse de utilização como reforço de matrizes termofixas e termoplásticas [ㅁ - $\underline{5}$ ]. Esse interesse está diretamente relacionado, entre outros fatores, à obtenção de materiais compósitos com aplicações tecnológicas na indústria automobilística, na construção civil e nas embalagens de alimentos.

No Brasil, as ocorrências de depósitos naturais de fibras de sílica amorfa (denotada por FNSA) são encontradas no fundo de lagoas e jazidas em diferentes regiões do sudeste, do nordeste e do centro-oeste. As fibras naturais de sílica amorfa são beneficiadas a partir do mineral espongilito, atóxica, densidade na faixa de 1,50 a 1,90 g.cm ${ }^{-3}$, elevada resistência à tração e módulo de elasticidade, sendo menos abrasivas do que as fibras de vidro [ $\underline{6}$ - 8]. Esses materiais são provenientes de precipitações biogenéticas de esponjas de água doce que utilizam os espículos como uma forma de suporte em sua estrutura, apresentando como elementos constituintes a sílica e pequenas quantidades de alumina, ferro, metais alcalinos e matéria orgânica, necessitando de beneficiamento.

Visando o aproveitamento de recursos naturais disponíveis em território nacional e buscando-se alternativas que sejam, economicamente, viáveis como reforço de matrizes poliméricas, compósitos de resina epóxi e fibras naturais de sílica amorfa (FNSA) foram preparados em diferentes composições e avaliado o comportamento tensão-deformação.

\section{MATERIAIS E MÉTODOS}

Fibras naturais de sílica amorfa (FNSA), sem e com modificação superficial com aminossilano, e as fibras de vidro (FV) foram gentilmente fornecidas pela Cerâmica São Caetano Ltda e Fiberglass ${ }^{\mathrm{R}}$, respectivamente. A resina epóxi do tipo éter diglicidílico do bisfenol A, DGEBA, (TCR 550) com o endurecedor do tipo trietileno tetramina, TETA, foram adquiridos através da Fiberglass ${ }^{\mathrm{R}}$.

As densidades das fibras de FNSA e FV foram determinadas por picnometria, utilizando água destilada e desgaseificada como solvente a $25^{\circ} \mathrm{C}$. Os valores obtidos correspondem à média de três medidas experimentais.

A modificação superficial das fibras de sílica amorfa, com aminossilano (FNSA-AS), foi realizada em duas etapas, conforme o método descrito na literatura [1]. Inicialmente, as fibras foram adicionadas numa solução de $\mathrm{HCl} 1 \mathrm{~mol} . \mathrm{L}^{-1}$ e mantidas sob agitação durante $2 \mathrm{~h}$ na temperatura de $60^{\circ} \mathrm{C}$, depois, lavadas com água destilada e secas sob vácuo por $48 \mathrm{~h}$. Em seguida, as fibras foram adicionadas em uma solução $2 \%$ de aminossilano em álcool etílico/água (90/10 v/v) e mantidas sob agitação à temperatura ambiente, por $3 \mathrm{~h}$. Posteriormente, as fibras foram lavadas com tolueno e secas em estufa por $12 \mathrm{~h}$, na temperatura de $60^{\circ} \mathrm{C}$.

Os compósitos foram preparados a partir da adição 6, 12, ou 17 \% em volume de FNSA ou FV, à mistura de resina/agente de cura na proporção de $50 / 50(\mathrm{~m} / \mathrm{m})$, mantendo-se sob agitação e vácuo durante 10 min, à temperatura ambiente. Os corpos-de-prova com dimensões padrões, estabelecidas pela Norma ASTMD-638-90, foram preparados a partir do vazamento da mistura em moldes de silicone.

Os ensaios de tensão-deformação foram realizados em máquina EMIC, utilizando-se uma célula de carga com capacidade de $1 \mathrm{kN}$, um extensômetro com fundo de escala de $250 \mathrm{~mm}$, uma resolução de $100 \mu \mathrm{m}$ e velocidade do travessão de $100 \mathrm{~mm} \cdot \mathrm{min}^{-1}$. Foram utilizados 10 corpos-de-prova para cada composição.

As fibras e a superfície de fratura dos corpos-de-prova, os quais foram recobertos com uma fina camada de ouro em um metalizador (ISI-D2 Diode Sputtering System), foram observadas em um microscópio eletrônico de varredura (MEV), modelo PHILIPS XL 30. A razão de aspecto (relação entre comprimento e diâmetro) das fibras foi determinada a partir da média de 100 medidas do comprimento e do diâmetro.

\section{RESULTADOS E DISCUSSÕES}

\subsection{Caracterização das fibras}

As fibras naturais de sílica amorfa apresentam extremidades afinadas e comprimento médio de $351,45 \pm 0,23 \mu \mathrm{m}$, determinado a partir das micrografias de microscopia eletrônica de varredura (Figura 1). As micrografias das fibras, com maior aumento, mostram a predominância de superfície lisa com textura uniforme e um furo central, espaço ocupado anteriormente pela silicateína [] $]$.

A análise de espectroscopia por energia dispersiva (EDS) mostrou que as FNSA são constituídas, predominantemente, de óxido de silício, sugerindo que o beneficiamento das fibras eliminou, completamente, a matéria orgânica e outros componentes constituintes da esponja de água doce. 


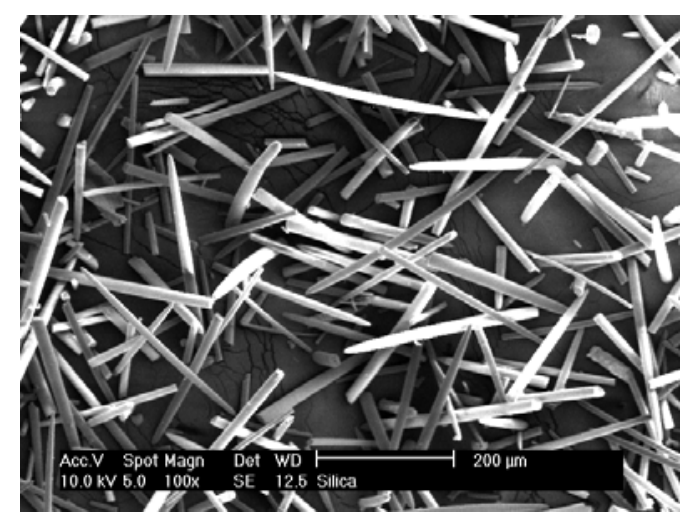

(a)

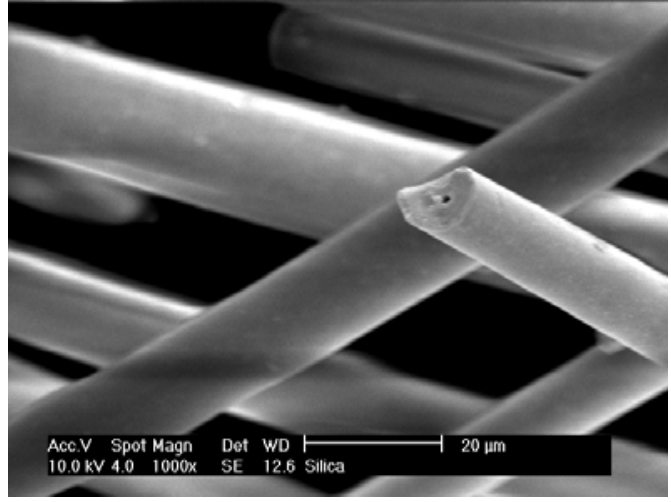

(b)

Figura 1: Micrografias de FNSA obtidas por microscopia eletrônica de varredura com aumentos de (a) 100 e (b)1000 vezes.

As razões de aspecto e as densidades das fibras naturais de sílica amorfa e das fibras de vidro utilizadas neste trabalho estão apresentadas na Tabela 1. Comparadas às fibras de sílica, as fibras de vidro apresentam menor relação aspecto e maior densidade. Portanto, para uma mesma fração volumétrica de fibras incorporadas na matriz polimérica, a quantidade em massa de FNSA será menor do que as FV.

Tabela 1: Valores da razão de aspecto (L/D) e densidade das FNSA e FV.

\begin{tabular}{c|c|c}
\hline Amostra & L/D & $\begin{array}{c}\text { Densidade } \\
\left(\mathrm{g} \cdot \mathrm{cm}^{-3}\right)\end{array}$ \\
\hline FV & $10,4 \pm 1,3$ & $2,49 \pm 0,13$ \\
\hline FNSA & $12,7 \pm 1,2$ & $1,75 \pm 0,11$ \\
\hline
\end{tabular}

\subsection{Caracterização dos compósitos de resina epóxi reforçados com fibras}

As micrografias das superfícies de fratura dos corpos-de-prova dos compósitos submetidos ao ensaio de tração, com 6 \% em volume de fibras naturais de sílica amorfa, mostram vazios devido ao arrancamento das fibras durante o ensaio de tração (Figura 2A). Na micrografia aumentada, podem ser melhor observadas as fibras fraturadas sem adesão com a matriz. A modificação química de superfície das fibras de sílica com aminossilano favorece a adesão interfacial entre fibra/matriz, conforme mostrado na Figura 2B. Essa diferença morfológica entre a utilização de fibras tratadas e não modificadas superficialmente está relacionada à interação química do grupo organo-funcional presente no agente de acoplamento com o grupo epóxi da matriz polimérica []].

Os compósitos com FV moídas ou FNSA possuem maiores valores de tensão na ruptura do que o polímero puro, evidenciando que a incorporação de fibras na matriz polimérica produz um aumento significativo em relação à capacidade de reforço da fibra (Figura 3). O valor da tensão na ruptura passou de 50 para $54 \mathrm{MPa}$, utilizando-se, como agente de reforço, $6 \%$ em volume de fibras tratadas superficialmente (FNSA-AS) em relação às FNSA não tratadas. Conforme descrito na literatura, o tratamento das fibras com aminossilano promove uma interação interfacial, resultando em uma maior transferência de tensão da matriz para a fibra e uma maior resistência à tração [1, $\underline{7}, \underline{8}]$. A diferença entre os valores da tensão na ruptura foi reduzida para percentagem, em volume de fibra, de 17,5 \%. O aumento da percentagem de fibras, também, reduziu a tensão na ruptura de todos os compósitos estudados, mantendo um maior valor para os compósitos com fibras tratadas superficialmente. Esse comportamento está relacionado à redução da percentagem de constituinte da matriz, o que induz um aumento do número de contato entre as fibras, conforme ilustrado nas micrografias da Figura 3, para as superfícies de fratura com percentagem em volume de $6 \%$ e $17,5 \%$ de FNSA. Portanto, o aumento da percentagem de fibras, na matriz, acarreta um aumento da concentração de tensão localizada no material, reduzindo a resistência à tração para todos os compósitos estudados. 

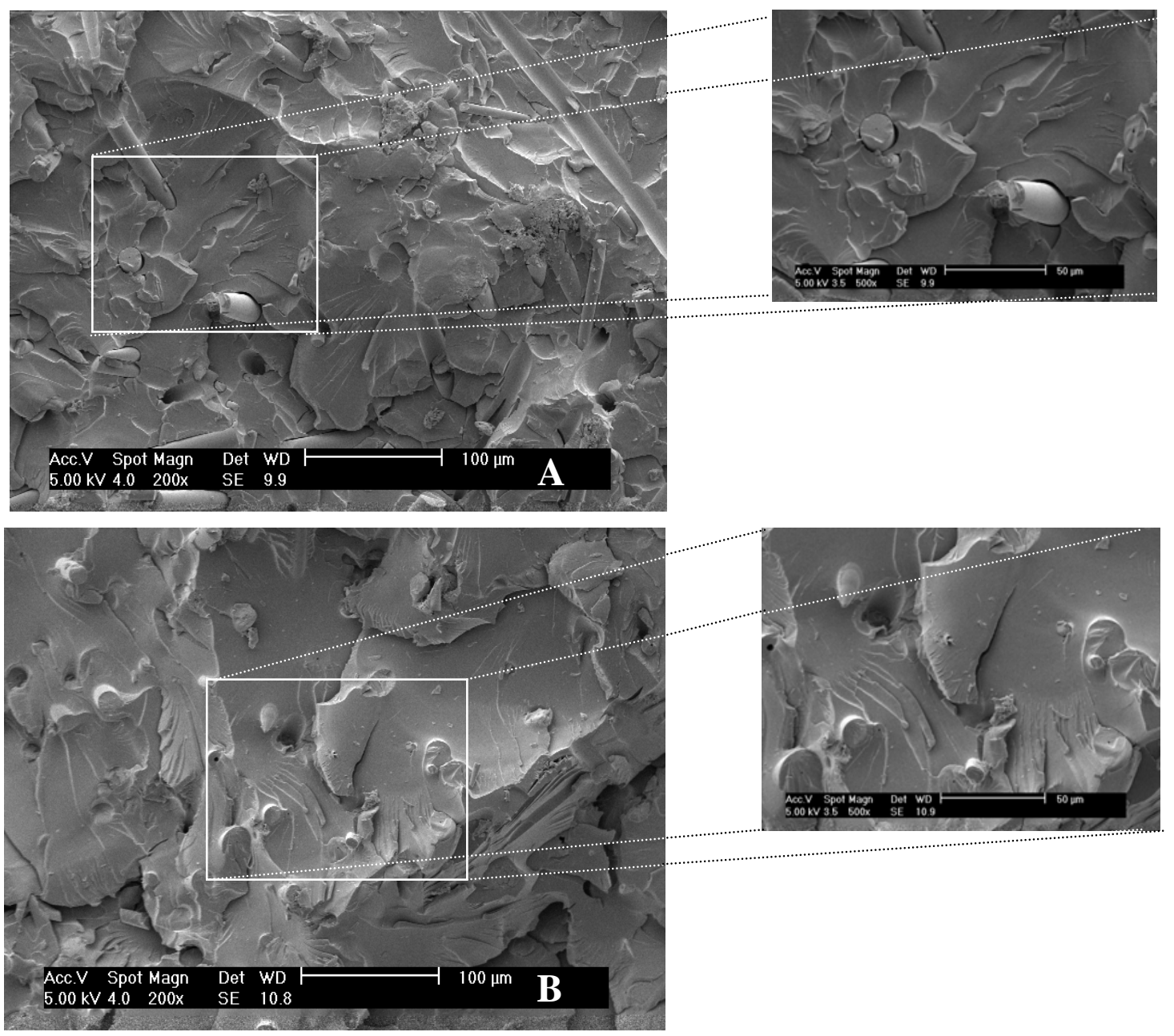

Figura 2: Micrografias das superfícies de fratura dos corpos-de-prova dos compósitos submetidos aos ensaios de resistência à tração: (A) Epóxi/FNSA e (B) Epóxi/FNSA-AS.

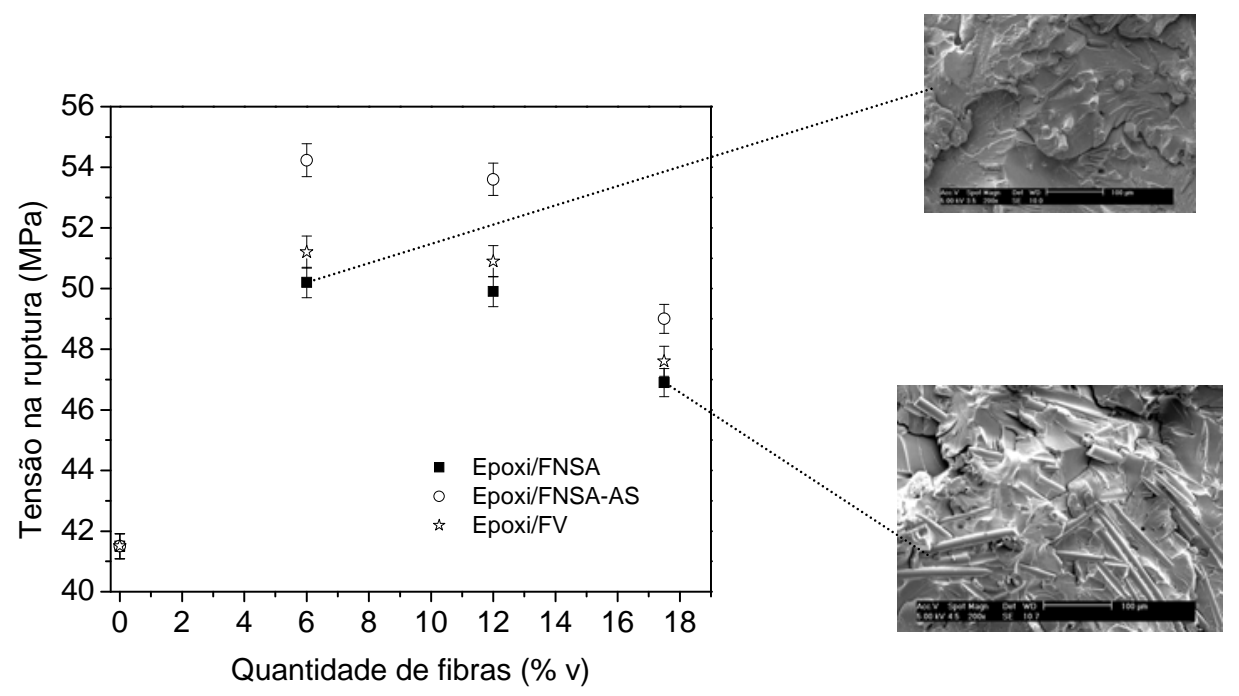

Figura 3: Gráfico da tensão na ruptura dos compósitos em função da quantidade de fibras. As micrografias mostram a superfície de fratura dos compósitos com 6\% e 17,5 \% de fibras de sílica amorfa não tratada. 
A Figura 4 apresenta os resultados de módulo de elasticidade e deformação na ruptura dos corposde-prova do polímero puro e compósitos. Os valores do módulo de elasticidade para os diferentes compósitos são da mesma ordem de grandeza, para as diferentes frações de volume de fibras. Por outro lado, a deformação na ruptura apresenta variação mais acentuada com aumento da percentagem de FNSA sem e com modificação superficial. Ocorre uma variação de 22 \% e 50 \% para quantidade de fibras naturais de sílica amorfa de 6 \% e 17,5 \% em volume, respectivamente, conforme ilustrado na Figura 4-B. A adesão entre as fases, avaliadas por microscopia eletrônica de varredura, resulta em maior transferência de tensão da matriz para as fibras, aumentando a resistência à tração e deformação do compósito.

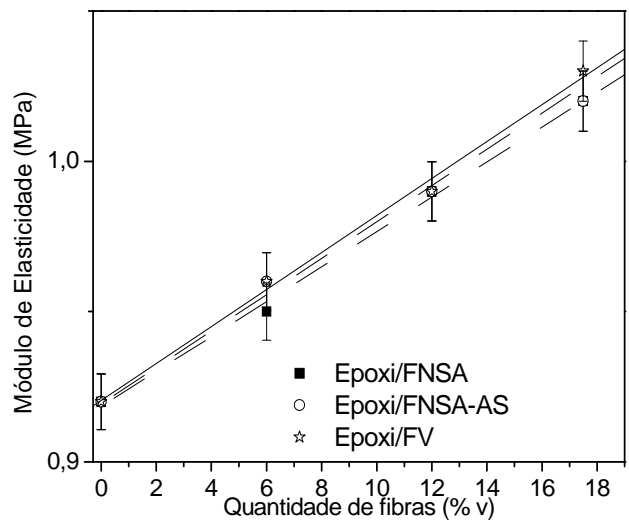

(a)

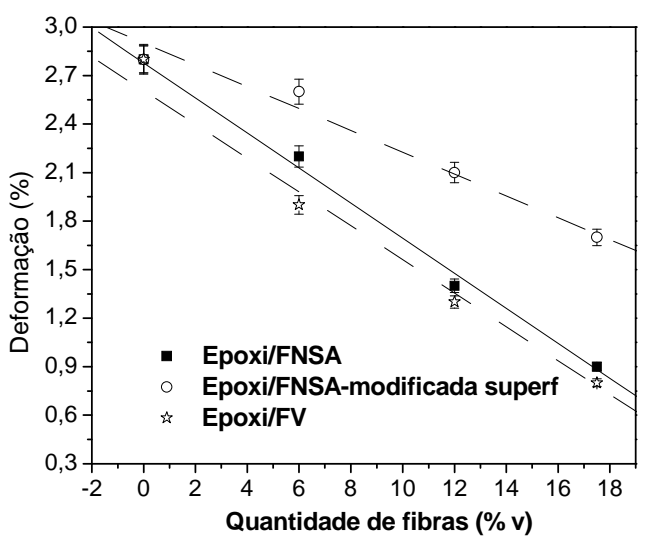

(b)

Figura 4: Curvas de deformação máxima e módulo de elasticidade, em função da quantidade de fibras.

\section{CONCLUSÕES}

Os ensaios de tração dos compósitos estudados sugerem que as fibras naturais de sílica amorfa são materiais viáveis de serem utilizadas como agente de reforço em matrizes de resina epóxi. Os compósitos de epóxi reforçados com FNSA sem modificação superficial apresentaram propriedades mecânicas de tração similares quando comparados com compósitos reforçados com fibras de vidro moídas, que são materiais tradicionalmente usados para reforço de polímeros. As FNSA com modificação superficial apresentaram desempenho superior em relação à utilização de fibras naturais de sílica amorfa sem tratamento e fibras de vidro.

As análises morfológicas das superfícies de fratura das amostras de epóxi reforçadas com FNSA com tratamento superficial mostraram adesão entre a fibra e matriz. A interação interfacial entre as fases no compósito fibra/matriz proporcionou um aumento na transferência de tensões da matriz para a fibra, aumentando significativamente a resistência à tração.

Concluindo, as fibras naturais de sílica amorfa podem ser utilizadas como agente de reforço em matriz de resina epóxi, com vantagens relacionadas às propriedades mecânicas, baixa densidade e ecologicamente aplicáveis, uma vez que são obtidas de fontes naturais e jazidas encontradas em diferentes regiões brasileiras.

\section{AGRADECIMENTO}

Os autores agradecem ao CNPq pelo apoio financeiro ao projeto de pesquisa.

\section{BIBLIOGRAFIA}

[1] DIBENEDETTO, A.T., "Tailoring of interfaces in glass fiber reinforced polymer composites: a review” Material Science and Engineering, v. 302, n. 1, pp. 74-82, 2001. 
[2] JOSEPH, K., MEDEIROS, E.S., CARVALHO, L.H., “Compósitos de matriz de poliéster reforçados por fibras curtas de sisal”, Polímeros: Ciência e Tecnologia, v. 9, n. 4, pp. 136-140, out/dez 1999.

[3] BLEDZKI, A.K., ZHANG, W., CHATE, A., "Natural-fibre-reinforced polyurethane microfoams", Composites Science and Technology, v. 61, n. 7, pp. 2405-2411, December 2001.

[4] RIALS, T.G., WOLCOTT, M.P., NASSAR, J.M., "Interfacial contributions in lignocellulosic fiberreinforced polyurethane composites”, Journal of Applied Polymer Science, v. 80, n. 4, pp. 546-555, 2001.

[5] AL-QURESHI, H.A., SILVA, J.L.G., "Mechanics of wetting system of natural fibers with polymeric resin”, Journal of Materials Processing Technology, v. 92, n. 93, pp. 124-128, August 1999.

[6] ESPER, J.A.M., Caracterização Mineralógica de Espongilito da Região de João Pinheiro - MG, Dissertação de M.Sc. em Engenharia de Minas, Universidade Federal de Minas Gerais, Belo Horizonte, Brasil, 2000

[7] SALIBA Jr., C.C., OREFICE, R.L., CARNEIRO, J.R.G., DUARTE, A.K., SCHNEIDER, W.T., FERNANDES, M.R.F., "Effect of the incorporation of a novel natural inorganic short fiber on the properties of polyurethane composites”, Polymer Testing, v. 24, n.7, pp. 819- 824, 2005.

[8] BARRA, G.M.O., FREDEL, M.C., AL-QURESHI, H. A., SALIBA Jr., C.C., TAYLOR, A., "Properties of chemically treated natural amorphous silica fibers as polyurethane reinforcement”, Polymer Composites, v. 27, n. 5, pp. 582-590, 2006. 